\title{
Preparation of Microcapsules and Half Life of the Kiwi Fruit Seed Oil by Complex Coacervation
}

\author{
Zhi Zhang1*, Mengting Pan ${ }^{1}$, Dongmin Liu² \\ ${ }^{1}$ Northeast Forestry University, Harbin, China \\ ${ }^{2}$ Dujiangyan Tianyi Kiwi Fruit Industrial Technology Institute, Dujiangyan, China \\ Email: *mlbsabc@sina.com
}

How to cite this paper: Zhang, Z., Pan, M.T. and Liu, D.M. (2017) Preparation of Microcapsules and Half Life of the Kiwi Fruit Seed Oil by Complex Coacervation. American Journal of Plant Sciences, 8, 191200.

http://dx.doi.org/10.4236/ajps.2017.82015

Received: December 12, 2016

Accepted: January 19, 2017

Published: January 22, 2017

Copyright $\odot 2017$ by authors and Scientific Research Publishing Inc. This work is licensed under the Creative Commons Attribution International License (CC BY 4.0).

http://creativecommons.org/licenses/by/4.0/ (c) (i) Open Access

\begin{abstract}
The experiment adopts complex coacervation to prepare microcapsules. Through the experimental comparison, soybean protein isolated-maltodextrin is determined as the wall material for the experimental preparation of the microcapsules of kiwi fruit seed oil. This paper researched the effects of wall material concentration, core wall ratio and other factors on complex coacervation of kiwi fruit seed oil microcapsules embedding rate, determining that the best wall material concentration is $1 \%$, core wall ratio is $1: 1$, and the optimum $\mathrm{pH}$ ratio is 3.0 , temperature is $40^{\circ} \mathrm{C}$, and the optimum curing time is 6 hours. The experiment carried out half life research on the microcapsules prepared by the complex coacervation of kiwi fruit seed oil microcapsule. By calculation: the degradation rate constant of kiwi fruit seed oil microcapsules prepared by complex coacervation is 2.793. According to the regression equation it can calculate the half life of kiwi fruit seed oil microcapsules is 18.58 months, about a year and a half.
\end{abstract}

\section{Keywords}

Kiwi Fruit Seed Oil Microcapsule, Complex Coacervation, Half Life

\section{Introduction}

Kiwi fruit seed oil has antioxidant, clearing free radicals inside the body, hypolipidemic, absorption of ultraviolet, anti-cancer, anti-tumor, sterilization, deodorant and other physiological activities. Due to the linolenic acid and linoleic acid and other unsaturated fatty acids, in the external environment, the kiwi fruit seed oil cannot exist stably and can be easily disturbed by the oxygen, water and other substances in the air, then gradually be oxidized and lose the original nu- 
tritional value [1]. In addition, due to the instability of physical and chemical properties of fatty acid, the sunlight in the external environment will lead to the aging and degeneration of lipid, the rancidity phenomenon, which not only affects the flavor and taste of kiwi fruit seed oil, but also produces certain poisonous side effect, bringing greatly inconvenience to the production technology of kiwi fruit seed oil.

Microcapsules can play a role in reducing the toxic and side effects of substances, shielding the bad taste and odor of the core material, changing the physical properties of the material, and controlling the release mode and process of the core material. Compared with other preparation methods of microcapsules, complex coacervation has the advantages of mild conditions, low requirements of equipment and simple operation [2]. The microcapsules products prepared by this method generally have excellent thermal stability, high humidity resistance and good controlled release properties.

\section{Test Methods}

\subsection{Method for Determination of Embedding Rate of Microcapsules}

Embedding rate is an important index to evaluate the quality of prepared microcapsules. The higher embedding rate of kiwi fruit seed oil microcapsules the better. Due to the influence of production process, the kiwi fruit seed oil as the core material cannot be completely wrapped in the wall material, some part of the oil will be exposed in the surface of microcapsules. The high embedding rate means the less amount of core material exposed in the surface of microcapsules, the less amount exposed to the external environment, so the products have higher stability and long storage time. Therefore, the embedding ratio is one of the important parameters in the production of microcapsules [3]. Measurement methods are from Han Lulu et al. Formula (1-1) [4]:

Embedding rate

$=\left(1-\frac{\text { The content of kiwi fruit seed oil on the microcapsu les surface } / \mathrm{g}}{\text { The total content of microcapsu les kiwi fruit seed oil } / \mathrm{g}}\right) \times 100 \%$

\subsection{Study on Preparation of Microcapsules of Kiwi Fruit Seed Oil by Complex Coacervation Method}

1) Test method of complex coacervation preparation

a) Preparation of mixed rubber solution

Evenly mix a certain amount of kiwi fruit seed oil and an appropriate amount of tween-80 emulsifier, and the diffluent gelatin in a certain proportion. After 5 min, uniformly mix the mixture of gelatin-kiwi fruit seed oil emulsion and wall material, gently stir the mixture to form emulsion. Then the mixture liquor is microcystic, the outer layer glue of glue soluting wraps kiwi fruit seed oil core material. Constantly stir, add $1 \%$ acetic acid solution into the solution to adjust the $\mathrm{pH}$ of mixture solution to 4.0 weak acid. 
b) Complex coacervation and curing of microcapsules

Drop the mixed evenly emulsion mixture solution to the beaker, with natural cooling to room temperature, place in ice water bath, 60 per minute after thermostatic mixing for $20 \mathrm{~min}$ with the stirring rate of 30 - 60 rings per minute, drop $10 \% \mathrm{NaOH}$ solution and to adjust the $\mathrm{pH}$ of mixture solution to about 8.5. In the whole process, keep stirring for $20 \mathrm{~min}$, so as to make composite wall material produce complete polymerization precipitation. When there is clean white solid precipitation in the beaker gradually, keep the mixture solution still standing for $4 \mathrm{~h}$.

c) The collection of microcapsules

Keep the microcapsules dispersion solution after finish curing still standing, filtration, wash the solid matter with small amount of distilled water for several times, put the microcapsules after filtration into the vacuum freeze dryer for drying, then sift the microcapsules through 200-mesh sieve to get kiwi fruit seed oil microcapsules products [4].

2) Effect of composite wall material composition on the embedding rate of kiwi seed oil microcapsules

This experiment selected four kinds of composite wall material to examine the influence of wall material types and proportion of wall materials on the embedding rate of microencapsules [5]. The amount of wall material $0.25 \mathrm{~g} / \mathrm{g}$, wall material concentration $1.0 \%, \mathrm{pH} 3.0$, core wall ratio of $1: 1$, the temperature of $40^{\circ} \mathrm{C}$, 6 hours of curing, then carry out microcapsule preparation. With the embedding rate as the index, the composite wall material was selected respectively as:

a) Gelatin and xanthan gum + sucrose: the ratio of gelatin and sucrose is 3:17, the additive amount of xanthan gum accounts for $0.3 \%$ of the amount of emulsion; the optimum additive amount of core material accounted for wall material is $20 \%$; the total solid content of emulsion is $20 \%$.

b) Isolated soybean protein + maltodextrin: isolated soy protein: maltodextrin as 1:1; core material: wall material as $1: 1$; the total solid content of emulsion is $20 \%$.

c) Arabic gum + maltodextrin + corn syrup:Arabic gum:maltodextrin, corn syrup as 22.5\%:25.0\%:52.5\%; core material:wall material as 1:1.

d) Arabic gum + maltodextrin + corn syrup + sucrose ester:Arabic gum:maltodextrin:corn syrup as 1:1:1, the additive amount of sucrose ester accounts for $0.8 \%$ of the amount of emulsion; the additive amount of core material accounts for $30 \%$ of wall material; the total solid content of emulsion is $20 \%$.

Respectively weigh and take the relevant materials according to the above groups into the beaker, add the appropriate amount of distilled water for full soaking, heat in water bath at a temperature about $80^{\circ} \mathrm{C}$, fully stir several wall materials to make it fully dissolved. Put the four solutions in the thermostat of $45^{\circ} \mathrm{C}$ for sealing preservation [6].

3) Effect of wall material concentration on the embedding rate of kiwi seed oil microcapsules

Refer to the best wall material of the above experimental screening. Prepare 
Microcapsules at the concentration of $0.5 \%, 1 \%, 1.5 \%, 2 \%, 2.5 \%, 3 \%$, with embedding rate as the indicators, to investigate the effect of wall material concentration on the micro encapsulation technology of kiwi fruit seed oil [7].

4) Effect of core wall ratio on the embedding rate of kiwi seed oil microcapsules

Refer to the best conditions of the above experimental screening. The addition ratio of core material and wall material is $1: 0.5,1: 1,1: 1.5,1: 2,1: 3$, with embedding rate as the indicators, to investigate the effect of core wall addition ratio on the microcapsules preparation technology of kiwi fruit seed oil.

5) Effect of solution $\mathrm{pH}$ value on the embedding rate of kiwi fruit seed oil microcapsules in the process of condensation

Refer to the best conditions of the above experimental screening. The emulsion $\mathrm{pH}$ is $2.0,3.0,4.0,5.0,6.0,7.0$, respectively in the process of condensation, with embedding rate as the indicators, to investigate the effect of solution $\mathrm{pH}$ value on the microcapsules preparation technology of kiwi fruit seed oil in the forming process of microcapsules [8].

6) Effect of temperature on the embedding rate of kiwi fruit seed oil microcapsules in the process of condensation

Refer to the best conditions of the above experimental screening. In the preparation process of microcapsules control the temperature at $20^{\circ} \mathrm{C}, 30^{\circ} \mathrm{C}, 40^{\circ} \mathrm{C}$, $50^{\circ} \mathrm{C}, 60^{\circ} \mathrm{C}$, with embedding rate as the indicators, to investigate the effect of temperature on the microcapsules preparation technology of kiwi fruit seed oil in the process of condensation.

7) Effect of curing time on the embedding rate of kiwi fruit seed oil microcapsules

Refer to the best conditions of the above experimental screening. After the condensation of microcapsules, control the curing time in $2 \mathrm{~h}, 4 \mathrm{~h}, 6 \mathrm{~h}, 8 \mathrm{~h}, 10 \mathrm{~h}$, $12 \mathrm{~h}$, with embedding rate as the indicators, to investigate the effect of curing time on the microcapsules preparation technology of kiwi fruit seed oil.

\subsection{The Retention Rate and Half Life of the Microcapsules of Kiwi Fruit Seed Oil}

Due to the influence of environmental factors in the storage process of microcapsules, the core material may be degraded and destroyed, and the most important indicator to determine the change of the stability is the retention rate of core material. Keep the kiwi fruit seed oil microcapsules prepared by complex coacervation in dark place at room temperature, take a sample and measure the content of kiwi fruit seed oil every 10 days and calculate the retention rate. The calculation of the retention rate of kiwi fruit seed oil is shown in the following Formula (1-2):

The retention rate of kiwi fruit seed oil (\%)

$=\frac{\text { The content of kiwi seedoil in the sample after storage for a period time }}{\text { The content of kiwi fruit seedoil in the sample before storage }} \times 100 \%$

Formula for calculating half life (1-3) 
Residual mass of kiwi seed oil microcapsules:

$$
m=m_{0} \times\left(\frac{1}{2}\right)^{\frac{t}{\tau}}
$$

Among them, $m$ refers to the residual mass; $m_{0}$ refers to the original mass; $t$ refers to decay time; $\tau$ refers to half life.

\section{Results and Discussion}

\subsection{Single Factor Test}

1) Effect of composite wall material composition on the embedding rate of the kiwi fruit seed oil microcapsules.

From the Table 1, four kinds of composite wall materials get the embedding rate in the same conditions of practice, and the embedding rate has no much difference. The embedding rate of compostie wall material of isolated soybean protein and maltodextrin has a slight advantage, that the embedding rate is relatively higher, the particle of microcapsule form is round and the size is uniform. In view of the low embedding rate of "gelatin + xanthan gum + sucrose" and other composite wall material composition, the bad particles of microcapsules and other shortcomings, ultimately determine isolated soybean protein and maltodextrin as the wall material to prepare kiwi fruit seed oil microcapsules, among which the ratio of isolated soybean protein and maltodextrin is 1:1.

2) Effect of wall material concentration on the embedding rate of kiwi seed oil microcapsules

Take isolated soybean protein-maltodextrin as the composite wall material, the quality ratio of $1: 1, \mathrm{pH}=4.0$, core wall ratio of $1: 1$, temperature of $40^{\circ} \mathrm{C}$, curing for 5 hours, carry out preparation of microcapsules, with embedding rate as the indicator, to investigate the effect of wall material concentration on the embedding rate of microcapsules. Results are shown in Figure 1.

From the figure, the concentration of wall material has a great influence on the embedding rate of encapsulation. When the wall material concentration is $1 \%$, the embedding rate is the best. Starting from $0.5 \%$, with the increase of the wall material concentration, the embedding rate of microcapsules increases firstly, then decreases slowly after the optimum concentration.

3) Effect of core wall ratio on the embedding rate of kiwi fruit seed oil microcapsules

Table 1. The effect of wall material composition on the embedding rate of microcapsules.

\begin{tabular}{ccc}
\hline Composite Wall Material Composition & Microcapsule's Embedding Rate & The Direct Observation On Microcapsules \\
\hline Gelatin + xanthan gum + sucrose & $65 \%$ & The powder and size is not uniform \\
Isolated soybean protein + maltodextrin & $80 \%$ & Round particles, uniform size, white color \\
Arabic gum + maltodextrin + corn syrup & $70 \%$ & Uniform size, but the powder is greasy \\
(d) Arabic gum + maltodextrin + corn syrup + sucrose ester & $72 \%$ & Round powder, uniform size
\end{tabular}




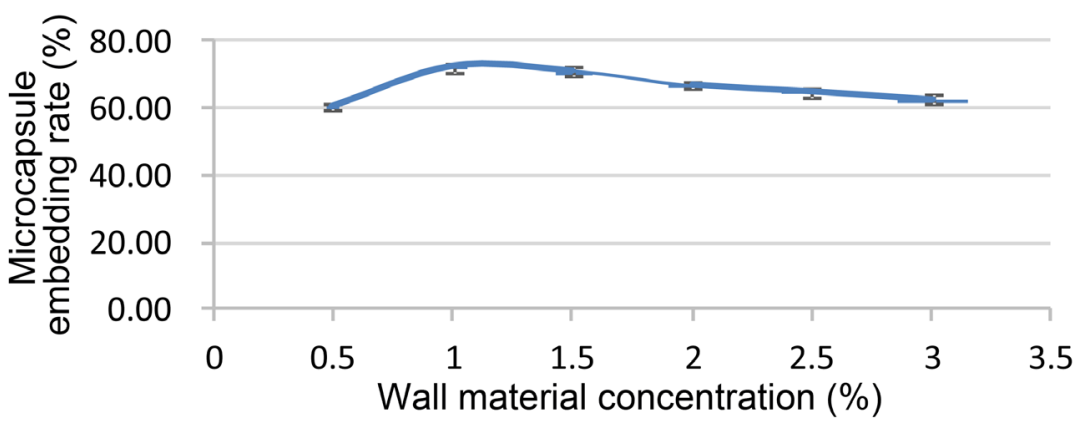

Figure 1. Effect of wall material concentration on the embedding rate of microcapsules.

Take isolated soybean protein-maltodextrin as the composite wall material, the quality ratio of $1: 1$, wall material concentration of $1 \%, \mathrm{pH}$ value of 4.0 , temperature of $40^{\circ} \mathrm{C}$, curing for 5 hours, carry out preparation of microcapsules, with embedding rate as the indicator, to investigate the effect of core wall ratio on the embedding rate of microcapsules. Results are shown in Figure 2 [9].

As shown in the figure, the embedding effect is the best when the core wall ratio is $1: 1$, up to $85 \%$. Both the overdose of core material and wall material will lead to the waste of material, which leads to the inevitable economic loss. Therefore, the appropriate choice of the core wall ratio is an important aspect of the research on the preparation process of microcapsules. The core material content is high, the wall material is not enough, leading to the waste of core material; the wall material is higher compared with core material, the core material content is small, leading to the waste of wall material. Thus, the ratio of the core wall is 1 : 1.

4) Effect of $\mathrm{pH}$ on the embedding rate of kiwi fruit seed oil microcapsules

Take isolated soybean protein-maltodextrin as the composite wall material, the quality ratio of $1: 1$, wall material concentration of $1 \%$, core wall ratio of $1: 1$, temperature of $40^{\circ} \mathrm{C}$, curing for 5 hours, carry out preparation of microcapsules, with embedding rate as the indicator, to investigate the effect of $\mathrm{pH}$ on the embedding rate of microcapsules. Results are shown in Figure 3.

From the above figure, within the range of $\mathrm{pH} 2-3$ in the mixture solution, the microcapsule products have high embedding rate and efficiency. When the $\mathrm{pH}$ of mixture solution deviates from 3, the embedding yield and efficiency shows a downward trend. This may be that at $\mathrm{pH} 3$, the electrochemical charge balance of gelatin ion and gum Arabic ion in the solution is good, when deviate from the value, the charge the two wall material ion changes, the positive electricity of isolated soybean protein reduces, the polymerization with the negative electricity of maltdextrin reduces, leading to the change of the polymer properties, and the decrease in the affinity with kiwi fruit seed oil, further resulting in the decrease of microencapsulation embedding rate. Thus the embedding rate is higher when $\mathrm{pH}=3$.

5) Effect of temperature on the embedding rate of kiwi fruit seed oil microcapsules

Take isolated soybean protein-maltodextrin as the composite wall material, 


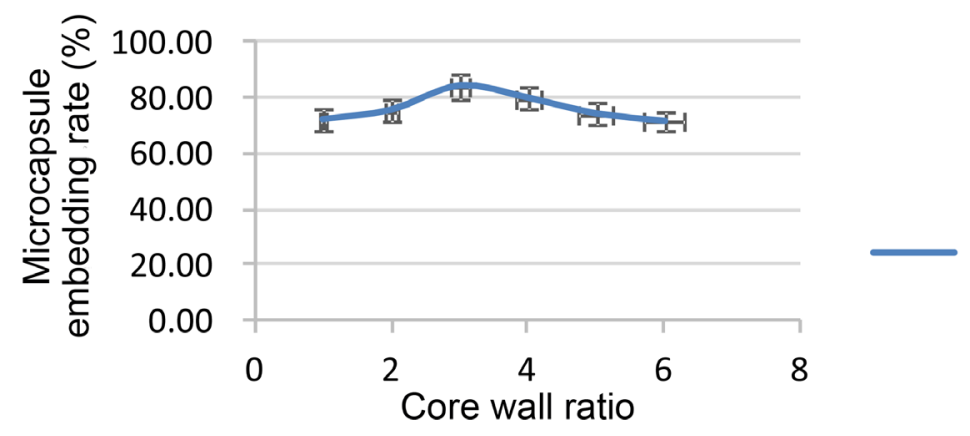

Figure 2. Effect of core wall ratio on the embedding rate of microcapsules.

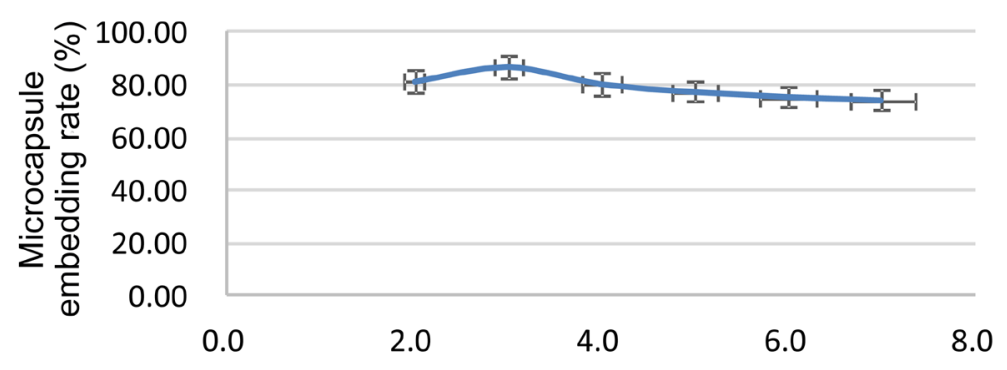

Figure 3. Effect of $\mathrm{pH}$ on the embedding rate of microcapsules.

the quality ratio of $1: 1$, wall material concentration of $1 \%$, core wall ratio of $1: 1$, $\mathrm{pH}=3.0$, curing for 5 hours, carry out preparation of microcapsules, with embedding rate as the indicator, to investigate the effect of temperature on the embedding rate of microcapsules. Results are shown in Figure 4.

From the above figure, with the increase of temperature, the embedding rate has a slight increase trend, when the temperature reaches $40^{\circ} \mathrm{C}$, the embedding rate decreases. As the temperature gradually increases, the protein hydration of isolated soybean protein-maltodextrin enhances, the effect of the complex condensation reaction strengthens, and the embedding rate increases. However, the high temperature will make the core material and water molecules move faster, the movement of small droplets in the reaction gradually enhances. So the temperature should be $40^{\circ} \mathrm{C}$.

6) Effect of curing time on the embedding rate of kiwi fruit seed oil microcapsules

Take isolated soybean protein-maltodextrin as the composite wall material, the quality ratio of $1: 1$, wall material concentration of $1 \%$, core wall ratio of $1: 1$, $\mathrm{pH}=3.0$, temperature of $40^{\circ} \mathrm{C}$, carry out preparation of microcapsules, with embedding rate as the indicator, to investigate the effect of curing time on the embedding rate of microcapsules. Results are shown in Figure 5.

As shown in the figure, at first with the increase of time, the embedding rate has a slight increase, when the time is $6 \mathrm{~h}$, the embedding rate reaches the highest, followed by the relatively flat embedding rate. Because the curing time is too short, the polymerization precipitation produced by the composite wall material is not complete; too long curing time will bring waste to the production, a 


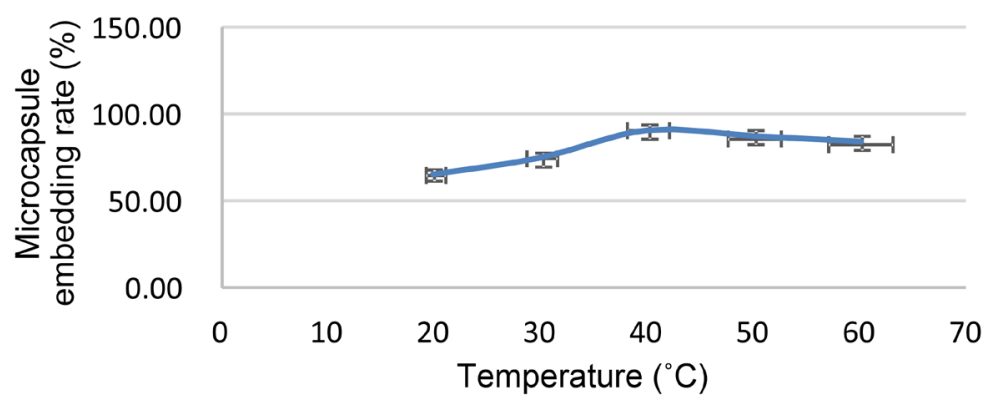

Figure 4. Effect of temperature on the embedding rate of microcapsules.

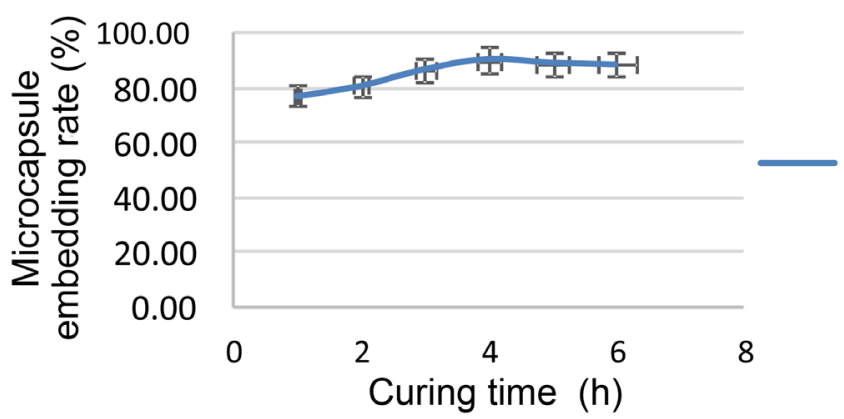

Figure 5. Effect of curing time on the embedding rate of microcapsules.

suitable time for the preparation of microcapsules has a very important significance.

\subsection{Study on the Preservation Rate of Kiwi Fruit Seed Oil Microcapsules}

Micro encapsulation through the embedding function, transforms the kiwi seed oil into the capsule form, wrapped in the wall material, which is a good solution to the problem of oxidation and deterioration of unsaturated fatty acids. Under the influence of external factors, the retention rate of microcapsules is an index to judge the passing of nutrients in the storage process of kiwi fruit seed oil, also a key factor to evaluate the quality of kiwi fruit seed oil microcapsules prepared by complex coacervation (Figure 6).

For the effect of external environment on the storage stability of composite condensed kiwi fruit seed oil microcapsules in natural environment, it can be seen that the retention rate of microcapsules decreases with the increase of storage time. At $20^{\circ} \mathrm{C}$, under a standard atmospheric pressure, the retention rate of the first three months is $99 \%, 95 \%, 93 \%$, respectively. With the extension of time, the retention rate also declines gradually. After storage for half a year and one year later, the retention rate is $82 \%$ and $65 \%$ respectively. Until after a year and a half, the retention rate of the kiwi seed oil microcapsules remains almost half of the original.

\subsection{Kinetic Parameter Analysis}

The test data was imported into the Excel software for analysis, and then the trend curve was added to get the regression analysis equation, so as to obtain the 


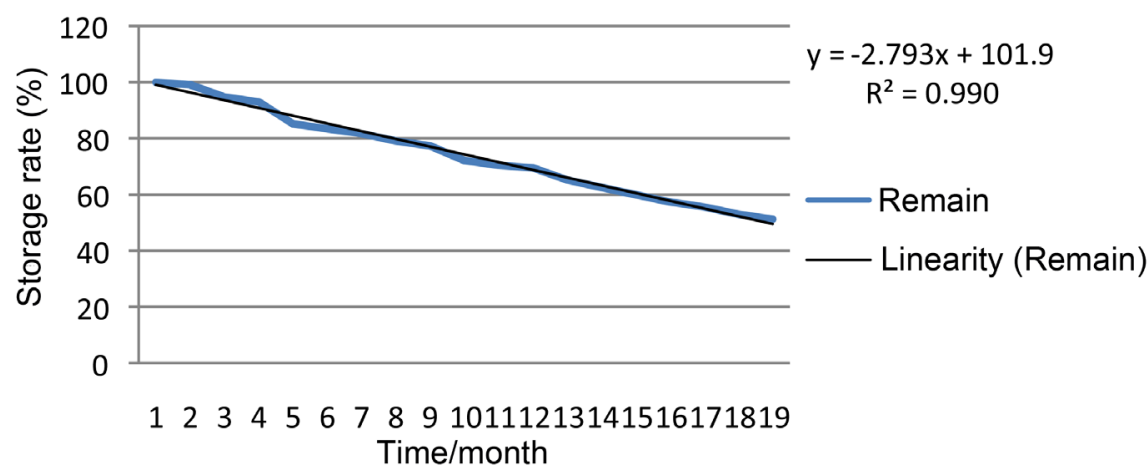

Figure 6. The preservation rate of composite condensed kiwi fruit seed oil microcapsules in the natural environment.

kinetic parameters through calculation (degradation rate constant and half-life). The related data is shown in the Table 2.

Under this test environment (NATURAL), the degradation rate constant of kiwi fruit seed oil microcapsules prepared by complex coacervation is 2.793. According to the regression equation, calculate the half-life of kiwi fruit seed oil microcapsules for 18.58 months, about a year and a half.

From the kinetic parameters analysis, it can explain: in the natural environment, light and oxygen and other external factors have an important effect on the storage of kiwi fruit seed oil, while complex coacervation microencapsulation has a good protection for the core material of kiwi fruit seed oil, which can reduce the opportunity of the light and oxygen exposure. To carry out the kinetic characteristics analysis of degradation rate etc., for microcapsule products is the quality control and one of the important indexes of the technological production of kiwi fruit seed oil microcapsule products.

\section{Conclusions}

In the research of process of kiwi fruit seed oil microcapsules prepared by complex coacervation, the screened optimum wall material ratio is isolated soybean protein-maltodextrin as 1:1. Single factor test was taken to determine the optimum process parameters: wall material concentration of $1 \%$, core wall ratio of $1: 1$, complex coacervation reaction temperature of $40^{\circ} \mathrm{C}, \mathrm{pH}$ value of 3 , the curing time of $6 \mathrm{~h}$. With embedding rate as response value, response surface regression test analysis was carried out, to determine the optimum process conditions: wall material concentration of $1.37 \%, \mathrm{pH}$ value of 2.8 , core wall ratio of $1: 1$. Under the optimum conditions, the theoretical maximum embedding efficiency is 93.98\% while the maximum embedding efficiency under the conditions through the experimental verification is $(93.64 \%+0.34 \%)$. Optimum experimental conditions were corrected to carry out experimental verification, with the wall material concentration of $1.37 \%, \mathrm{pH}$ of 3 , core wall ratio of 1:1, 3 times of verification, to get the average embedding efficiency of $93.78 \%$.

In the natural environment, we research the half life of kiwi fruit seed oil microcapsules prepared by complex coacervation. It can be seen that the retention 
Table 2. The kinetic constant of degradation of kiwi fruit seed oil microcapsules prepared by complex coacervation in the natural environment.

\begin{tabular}{ccccc}
\hline Sample & The Degradation Rate Constant & The Half-Value Period (Month) & $\mathrm{R}^{2}$ & Regression Equation \\
\hline Kiwi fruit seed oil microcapsules & 2.793 & 18.58 & 0.990 & $\mathrm{y}=-2.793 \mathrm{x}+101.9$ \\
\hline
\end{tabular}

rate of the core material of microcapsules decreases with the extension of storage time. At $20^{\circ} \mathrm{C}$, under a standard atmospheric pressure, with the extension of time, the retention rate also declines gradually. Until after a year and a half, the retention rate of the kiwi seed oil microcapsules remains almost half of the original. Through analysis of the kinetic data, the degradation rate constant of kiwi fruit seed oil microcapsules, prepared by complex coacervation for 2.793 , was calculated. According to the regression equation, the half-life of kiwi fruit seed oil microcapsules was calculated for 18.58 months, about a year and a half.

\section{Fund Project}

Jointly funded by "Heilongiiang province forest economic resources research, development and utilization collaborative innovation center" project and "excellence in agriculture and forestry personnel education" and training funding scheme reform pilot project (41110211).

\section{References}

[1] Gao, Y. (2012) Study on Preparation of Capsaicin Microcapsules by Complex Coacervation Process. Thesis, Changsha University of Science and Technology, Changsha.

[2] Wang, S.H., Zhang, H., Duan, C.J. and Zhang, J. (2006) Preparation Technology and Its Application of Microcapsules. Materials Review, S2, 376-379.

[3] Wang, K., Lin, S.L., Cheng, J. and Yang, Z.R. (2012) New Progress of Microcapsules Technology. Polymer Materials Science and Engineering, No. 5, 179-182.

[4] Han, L.L., Bi, L.W., Zhao, Z.D. and Li, D.W. (2011) Research Progress on Preparation Methods of Microcapsules. Biomass Chemical Engineering, No. 3, 41-46.

[5] Xie, Y.T. and Liu, X.G. (2005) Comparison of Preparation Methods of Microcapsules. Cereal and Food Industry, No. 1, 28-31.

[6] Han, L., Lv, C.M., Zhao, M.H., Zheng, P. and Liu, C. (2013) Preparation of Apple Kiwi Fruit Seed Oil Microcapsules by Complex Coacervation. Food Science, 20, 342-346.

[7] Han, G.T. (2013) Study on Preparation and Performance of Patchouli Oil Microcapsules. Thesis, Huazhong Agricultural University, Wuhan.

[8] Li, M. (2009) Separation and Micro Encapsulation of Linoleic Acid from Safflower Seed Oil. College of Food Science and Technology, Jiangnan University, Wuxi.

[9] Li, Y. and Pang, L.Y. (2009) Research Progress of Microcapsules Technology in Food Industry. Guizhou Agricultural Science, No. 11, 35-38. 
Submit or recommend next manuscript to SCIRP and we will provide best service for you:

Accepting pre-submission inquiries through Email, Facebook, LinkedIn, Twitter, etc. A wide selection of journals (inclusive of 9 subjects, more than 200 journals)

Providing 24-hour high-quality service

User-friendly online submission system

Fair and swift peer-review system

Efficient typesetting and proofreading procedure

Display of the result of downloads and visits, as well as the number of cited articles Maximum dissemination of your research work

Submit your manuscript at: http://papersubmission.scirp.org/

Or contact ajps@scirp.org 